\title{
CONFIRMATION OF DEOXYNIVALENOL PRESENCE IN CHILEAN WHEAT BY HIGH-PERFORMANCE THIN-LAYER CHROMATOGRAPHY-MASS SPECTROMETRY
}

\author{
MARIO VEGA-HERRERA ${ }^{1}$, RICARDO MADARIAGA², MARIO ARANDA' AND GERTRUD E. MORLOCK ${ }^{3 *}$ \\ ${ }^{1}$ Department of Food Science and Technology, Faculty of Pharmacy, University of Concepcion, \\ Barrio Universitario s/ $n$ Casilla 237, PO 403-0249 Concepcion, Chile. \\ ${ }^{2}$ INIA-Quilamapu, Casilla 426 Chillan, Chile. \\ ${ }^{3} J u s t u s$ Liebig University Giessen, Institute of Nutritional Science, Heinrich-Buff-Ring 26-32, 35392 Giessen, Germany.
}

ABSTRACT

Deoxynivalenol (DON) is a mycotoxin that belongs to trichothecene subgroup B. Due its detrimental effects for human health, DON has been analyzed and detected by nearly all chromatographic methods. The present work reports for the first time the application of HPTLC/MS to confirm the presence of DON in wheat crops. Chromatography was performed on silica gel $60 \mathrm{~F}_{254}$ HPTLC plates using toluene - ethyl acetate - formic acid 6:3:1 $(V / V / V)$ as mobile phase. After post-chromatographic derivatization with $10 \% \mathrm{AlCl}_{3}$, fluorescence detection was carried out at UV $366 />400 \mathrm{~nm}$. Bands identity and purity was confirmed by mass spectrometry acquiring analytes directly from the sample bands by means of TLC/MS elution head-based interface. Both, sample and standard bands showed clear mass signals at $m / z 341$ that corresponds to the formate adduct of DON [M+HCOO]. Thus, the presence of DON in the Chilean wheat sample was confirmed.

Keywords: Deoxynivalenol, mycotoxins, HPTLC/MS, Fusarium, TLC.

\section{INTRODUCTION}

Mycotoxins are secondary metabolites produced by several fungus genera, of which Aspergillus, Penicillium and Fusarium are the most important ones. Among other mycotoxins the genera Fusarium produces trichothecenes, which is one of the major class of mycotoxins composed of more than 200 toxins ${ }^{1}$. They are chemically classified in four groups (type A, B, C and D) according to the type of substitution of the base structure (tricyclic 12,13-epoxytrichothec9-ene). Deoxynivalenol (DON, Figure 1) is a type B trichothecene (containing a carbonyl group at $\mathrm{C} 8$ ) reported as one of the most prevalent food-associated mycotoxin, especially in cereal grains ${ }^{2}$. US and European studies have reported that more than $50 \%$ of cereal and cereal-derived samples are positive for $\mathrm{DON}^{2}$ and some of them with high concentrations ${ }^{3}$. Further, this mycotoxin is thermoresistant (up to $350^{\circ} \mathrm{C}$ ) being therefore stable during processing, e.g. cooking ${ }^{2}$. DON, also known as "vomitoxin" due to the emetic effect in pigs, produces relevant adverse (toxic) effects in humans. The alterations over the immune, gastrointestinal, endocrine and reproductive systems $\mathrm{s}^{2 ; 3}$ led to the Joint FAO/ WHO Expert Committee on Food Additives (JECFA) to establish a provisional maximum tolerable daily intake (PMTDI) of $1 \mu \mathrm{g} / \mathrm{kg}$ body weight for DON and its acetylated derivatives (3-Ac-DON and 15-Ac-DON) ${ }^{4}$. Regarding carcinogenicity, the International Agency for Research on Cancer (IARC) classified DON into Group $3^{5}$. In view of its toxic potential, the European Community ${ }^{6}$ established maximum levels for unprocessed and processed cereals $\left(200\right.$ to $1750 \mu \mathrm{g} \mathrm{kg}^{-1}$ ), which has been used to routinely evaluate its presence in several countries finding samples that exceed the proposed limits? . DON determination can be done by several analytical methods, including almost all chromatographic techniques, e.g. high performance liquid chromatography (HPLC) coupled to ultraviolet (UV) ${ }^{8}$, fluorescence (FL) ${ }^{9}$ and mass spectrometry (MS) ${ }^{10}$ detectors; gas chromatography (GC) coupled to $\mathrm{MS}^{11}$, flame ionization ${ }^{12}$ and electron capture ${ }^{13}$ detectors and high performance thin layer chromatography (HPTLC) with FL detection (FLD) ${ }^{14}$. These methods use an extraction procedure that generally includes a clean-up step with QuEChERs ${ }^{15}$, immunoaffinity ${ }^{16}$ or multifunctional solid phase extraction (SPE) columns ${ }^{17}$.

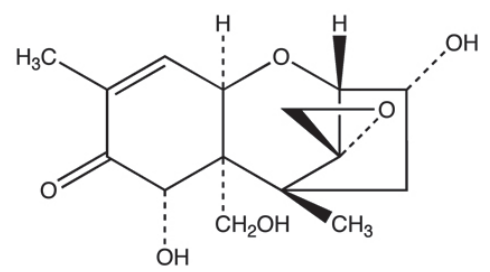

Figure 1. Structure formula of 4-deoxynivalenol (DON) [12,13-epoxy3,7,15-trihydroxytrichothec-9-en-8-one].
Many reports about cereal contamination with Fusarium toxins were published worldwide; whereas in Chile, located in the south-west of South America, there was no data about any outbreak attributable to Fusarium toxins present on domestic cereal production. First in February 2004, a fusariosis outbreak infecting a wheat crop was detected in the central part of the country. The wheat presented the classical symptoms of white head blight.

In this study, representative wheat crop samples were collected directly from the infected field as well as an overall sample was taken at the end of the harvest. A simple extraction procedure was used based on multifunctional SPE columns. These extracts were analyzed by a validated HPTLC/FLD method. Potential positive findings were confirmed by HPTLC/MS.

\section{EXPERIMENTAL}

\subsection{Chemicals and reagents}

Deoxynivalenol (DON) was purchased from Sigma (St. Louis, MO, USA). HPTLC plates silica gel $60 \mathrm{~F}_{254}(10 \times 10 \mathrm{~cm})$, aluminum chloride 6-hydrate, methanol, ethanol, toluene, ethyl acetate, formic acid and acetonitrile were obtained from Merck (Darmstadt, Germany). All solvents were of HPLC quality or distilled before its use. Ultra-pure water $(18 \mathrm{M} \Omega \mathrm{cm})$ was produced by Synergy System (Millipore GmbH, Schwalbach, Germany). Romer Labs Mycosep DON multifunctional columns 225 were obtained from Coring System Diagnostix (Gernsheim, Germany). Before usage, the plates were prewashed with methanol and dried for $30 \mathrm{~min}$ at $120^{\circ} \mathrm{C}$.

\subsection{Preparation of the standard solution}

A methanolic stock solution was prepared from the crystalline DON (100 $\left.\mu \mathrm{g} \mathrm{mL}^{-1}\right)$ and diluted 1:4 with methanol to obtain the standard solution $(25 \mu \mathrm{g}$ $\mathrm{mL}^{-1}$ ) stored at $-20^{\circ} \mathrm{C}$.

\subsection{Sample preparation}

Due to the matrix complexity and the expected low levels of DON ( $\mu \mathrm{g} /$ $\mathrm{kg}$ to $\mathrm{mg} / \mathrm{kg}$ ), an exhaustive sample preparation was required to accomplish an adequate clean up and analyte concentration. DON was extracted applying the method reported by Radova et al. ${ }^{18}$ with slight modifications. Briefly, wheat crop samples were ground in a Romer mill (Romer Labs, Union, MO, USA) to obtain a coarse grained powder. The powder $(25 \mathrm{~g})$ was mixed with $100 \mathrm{~mL}$ acetonitrile and water, 21:4 (V/V) by an Ultra-turrax T 25 (Janke and Kunkel, IKA Labortechnik, Staufen, Germany) at high speed for $5 \mathrm{~min}$. The solution obtained was filtered through a filter paper (MN-615, Macherey-Nagel, Düren, Germany). The filtrate ( $8 \mathrm{~mL}$ ) was transferred to an assay tube and the Mycosep DON multifunctional column 225 was pushed over it. The purified extract (4 $\mathrm{mL}$ ) was transferred to a vial and the solvent was evaporated to dryness under a gentle stream of nitrogen. The residue was dissolved in $500 \mu \mathrm{L}$ of ethyl acetate and methanol, 19:1 $(V / V)$. 


\subsection{Chromatography}

Sample extracts and standard solutions were applied with the Automatic TLC Sampler 4 (ATS 4, CAMAG, Muttenz, Switzerland) with the following settings: band length $6.0 \mathrm{~mm}$, dosage speed $150 \mathrm{~nL} \mathrm{~s}^{-1}$, distance from the left side $15.0 \mathrm{~mm}$ and from the lower side $8.0 \mathrm{~mm}, 10$ tracks per plate. For MS recordings, the application volumes were $12 \mu \mathrm{L}$ standard solution (300 $\mathrm{ng} /$ band) and $8 \mu \mathrm{L}$ sample solution (resulting in a similar concentration). Chromatography was performed in a Horizontal Development Chamber, 10 x $10 \mathrm{~cm}$ (CAMAG), with toluene - ethyl acetate - formic acid 6:3:1 (V/V/V) as solvent system. After 2 min drying in a stream of warm air, the plate was immersed in a solution of $10 \% \mathrm{AlCl}_{3}$ in ethanol - water $1: 1(\mathrm{~V} / \mathrm{V})$ using the TLC Immersion Device (CAMAG) and heated at $120^{\circ} \mathrm{C}$ on the TLC Plate Heater (CAMAG) for $10 \mathrm{~min}$. Fluorescence measurement of DON was performed at UV 366/ $>400 \mathrm{~nm}$ with the TLC Scanner 3 (CAMAG) using a slit dimension of $5.0 \mathrm{~mm} \times 0.3 \mathrm{~mm}$. All automated instruments were controlled via the software winCATS 1.4.1 Planar Chromatography Manager (CAMAG).

\subsection{Mass spectrometry}

For recording of mass spectra the underivatized plate was measured at $220 \mathrm{~nm}$ and the migration distance of each compound was marked with a soft pencil. Using an HPLC pump (HP 1100, Agilent Technologies, Palo Alto, USA) and the ChromeXtraktor interface (ChromAn, Holzhausen, Germany), the zone of interest on the HPTLC plate was directly eluted into the electrospray ionization (ESI) source of the VG platform II single-quadrupole mass spectrometer (Micromass, Manchester, United Kingdom). The zone of interest was sealed with the cutting edge of the elution head and eluted from the layer with a mixture of methanol and formate buffer $\left(10 \mathrm{mmol} \mathrm{L}^{-1}, \mathrm{pH} 4.0\right)$, 19:1 $(V / V)$, at a flow rate of $0.1 \mathrm{~mL} \mathrm{~min}^{-1}$. The MS system was operated in the full scan mode with the following parameters for ESI in the negative ionization mode: source temperature $120^{\circ} \mathrm{C}$; capillary voltage $-3.5 \mathrm{kV}$; $\mathrm{HV}$ lens $0.5 \mathrm{kV}$; cone voltage $-55 \mathrm{~V}$; dwell time $0.5 \mathrm{~s}$; inter channel delay 0.02 ; repeats 1 ; span 0.5. Data were processed with Mass Lynx 3.2 software (Micromass).

\section{RESULTS AND DISCUSSION}

\subsection{Method validation}

The validation of the method was carried out according to the guideline of the International Conference on Harmonization $(\mathrm{ICH})^{19}$ (Table 1). The regression analysis was performed with six DON concentration levels in triplicate. The recovery was calculated via three separate blank samples, each spiked at the concentration level of $1 \mathrm{mg} \mathrm{kg}^{-1}$. As indicator for the precision of the method, the repeatability of the sample analysis was determined. The limits of detection and quantification were calculated using a signal to noise ratio of 3 and 10 , respectively.
Table 1. Validation data for determination of DON in wheat by HPTLC/ FLD.

\begin{tabular}{|c|c|}
\hline Linearity $\left[\mathrm{ng} \mathrm{band}{ }^{-1}\right]$ & $8-120$ \\
\hline Equation by peak area & $y=16.08 x+23.12$ \\
\hline $\mathrm{r}$ and ${ }^{2}$ & 0.9991 and 0.9982 \\
\hline Recovery $\left[\%, n=3,1 \mathrm{mg} \mathrm{kg}^{-1}\right]$ & $90.1 \pm 6.4$ \\
\hline Repeatability $(\% R S D)^{\mathrm{a}}$ & 7.1 \\
\hline Detection limit $\left[\mathrm{mg} \mathrm{kg}^{-1}\right]^{\mathrm{a}}$ & 0.05 \\
\hline Quantification limit $\left[\mathrm{mg} \mathrm{kg}^{-1}\right]^{\mathrm{a}}$ & 0.19 \\
\hline
\end{tabular}

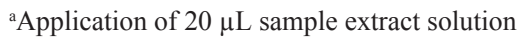

\subsection{Sample analysis}

Applying the validated method, representative wheat crop samples sampled directly from the field were analyzed. The overall wheat sample (sampled at the end of the harvest of 2004) was re-analyzed in 2005 and the mean value of DON $(n=3)$ was established to be $5.5 \pm 0.3 \mathrm{mg} \mathrm{kg}^{-1}(\% R S D$ $5.1 \%$ ) via peak height and $5.4 \pm 0.3 \mathrm{mg} \mathrm{kg}^{-1}(\% R S D 5.2 \%)$ via peak area. These values were in good agreement with the previous results determined directly after the harvest of 2004. Using the described chromatographic system and the Mycosep DON multifunctional column 225, the analyte (DON) was completely separated from the matrix. The proof of this specificity in detection can be clearly seen in the chromatogram illuminated at UV 366 nm (Figure 2).

\subsection{Confirmation by HPTLC/MS}

The presence of DON in the wheat sample was confirmed by HPTLC/ ESI-MS. The elution head-based ChromeXtraktor interface ${ }^{20}$, which reliability was already proven in trace analysis ${ }^{21 ; 22}$ and which was modified for elution from glass-backed HPTLC plates ${ }^{23}$, was a highly targeted tool to combine HPTLC with MS. Only from the selected zone of interest, mass selective compound information was obtained. The targeted use of HPTLC/MS avoided the automatic transfer of background signals and matrix signals into the MS system, which is the routine case for column-based hyphenations. Full scan mass spectra were recorded in the negative ionization mode between $\mathrm{m} / \mathrm{z} 200$ and 500 (Figure 3). High amounts of DON (300 $\left.\mathrm{ng} \mathrm{band}^{-1}\right)$, which corresponded to the sample zone intensity, were selected for direct elution into the MS.

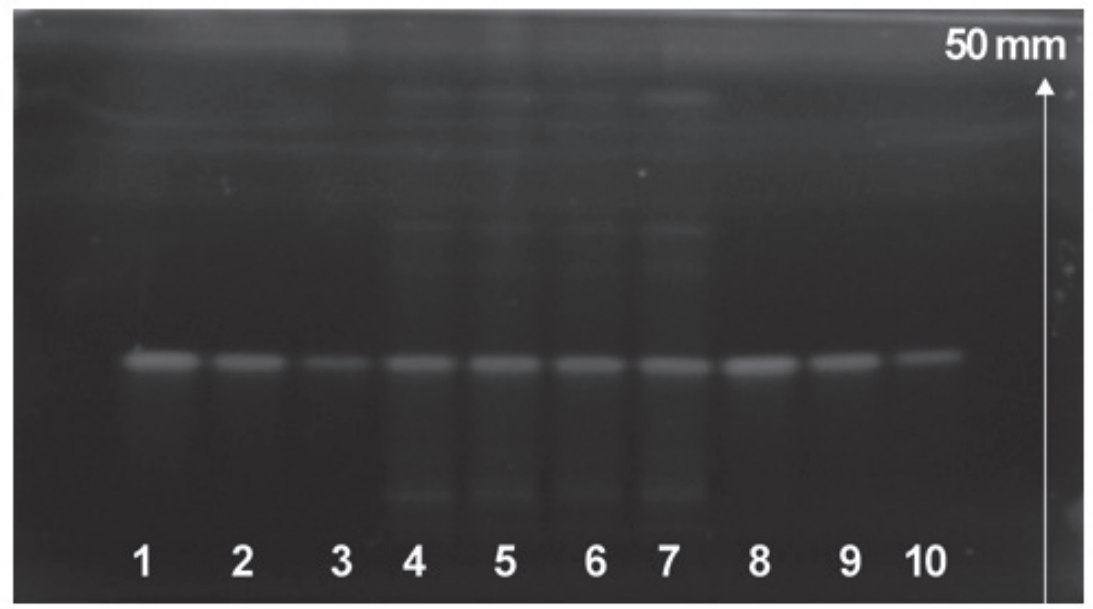

Figure 2. HPTLC chromatogram documented at UV $366 \mathrm{~nm}$ showing DON standard solutions (tracks 1-3 and 8-10: 75, 50 and $25 \mathrm{ng} \mathrm{band}^{-1}$; twofold determination), the Chilean whea sample containing DON (tracks 4-6) and the same sample extracted with a re-used Mycosep DON multifunctional column 225 (track 7). 


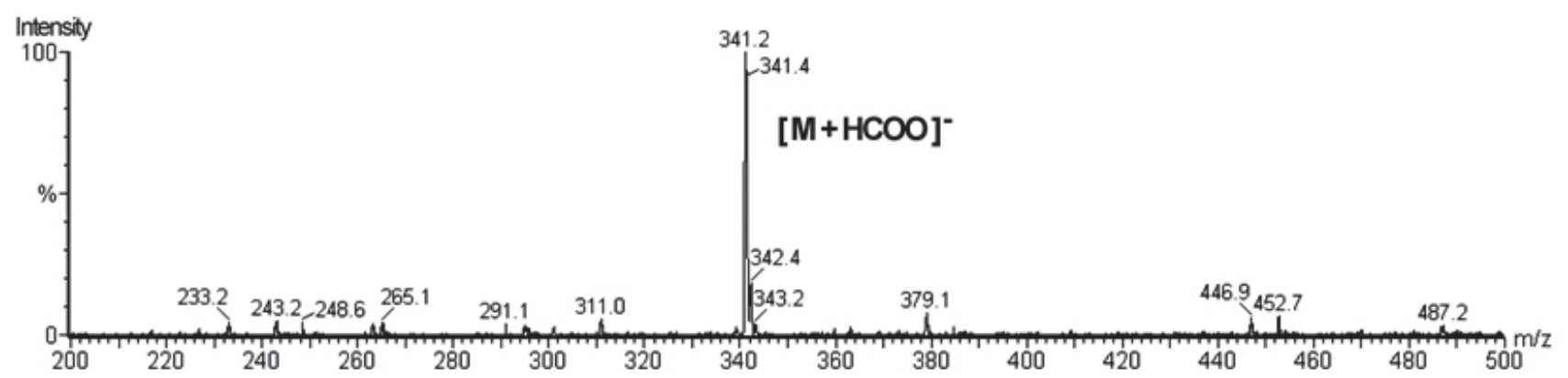

Figure 3. Confirmation by HPTLC/MS via direct elution from the HPTLC plate silica gel 60, exemplarily showing full scan mass spectrum of the 300 ng band ${ }^{-1}$ DON standard zone ionizing as formate adduct [M+HCOO] at $m / z 341$.

\section{CONCLUSIONS}

The outlined HPTLC/FLD/ESI-MS method for determination of DON in wheat offered a fast, low-cost alternative due to the coupling of an efficient screening method with a selective detector, if required. The confirmation of the results by HPTLC/MS avoided the assignment of false positives.

\section{ACKNOWLEDGEMENTS}

Thanks to Dr. Heinrich Luftmann, University of Münster, Germany, for providing the ChromeXtractor, to the Chilean Government as well as the University of Concepcion, Chile, (MYCOTOX project No. INCO Dev. ICA-CT 2002-10043) for financial support, to Merck, Darmstadt, Germany, and CAMAG, Muttenz, Switzerland for support regarding plate material and instrumental equipment.

\section{REFERENCES}

1.- S. P. McCormick, A. M. Stanley, N. A. Stover, N. J. Alexander. Toxins 3, 802, (2011)

2.- M. Maresca. Toxins 5, 784, (2013).

3.- J. J. Pestka. Arch Toxicol 84, 663, (2010).

4.- FAO/WHO (2011) Evaluation of certain contaminants in food: Seventysecond report of the Joint FAO/WHO Expert Committee on Food Additives.

5.- WHO/IARC (1993) Some Naturally Occurring Substances: Food Items and Constituents, Heterocyclic Aromatic Amines and Mycotoxins in IARC Monographs on the Evaluation of Carcinogenic Risks to Humans vol 56.
6.- European Commission. Official Journal of the European Union L364, 5, (2006).

7.- J. Sun, Y. Wu. Food Control 69, 90, (2016).

8.- A. Skendi, M. N. Irakli, M. D. Papageorgiou. Journal of Separation Science 39, 1425, (2016).

9.- M. Muscarella, M. Iammarino, D. Nardiello, C. Palermo, D. Centonze. Talanta 97, 145, (2012).

10.- Y. Liu, Y. Lu, L. Wang, F. Chang, L. Yang. Food Chem 197, Part B, 1271, (2016).

11.- L. Escrivá, L. Manyes, G. Font, H. Berrada. Food Additives \& Contaminants: Part A 33, 329, (2016).

12.- Z. H. Shar, G. A. Sumbal, S. T. H. Sherazi, M. I. Bhanger, S. M. Nizamani Chromatographia 77, 337, (2014).

13.- A. Hallier, F. Celette, J. Coutarel, C. David. Food Addit Contam Part A-Chem 30, 2159, (2013).

14.- S. Mishra, K. M. Ansari, P. D. Dwivedi, H. P. Pandey, M. Das. Food Control 30, 549, (2013).

15.- V. L. Pereira, J. O. Fernandes, S. C. Cunha. Food Chem 182, 143, (2015).

16.- S. Y. Jung, B. C. Choe, E. J. Choi, H. J. Jeong, Y. S. Hwang, G. Y. Shin, J. H. Kim. Food Sci Biotechnol 24, 1193, (2015).

17.- J. Rubert, Z. Dzuman, M. Vaclavikova, M. Zachariasova, C. Soler, J. Hajslova. Talanta 99, 712, (2012).

18.- Z. Radová, K. Holadová, J. Hajšlová. J Chromatogr A 829, 259, (1998).

19.- International Conference on Harmonisation (ICH). ICH Harmonised tripartite guideline validation of analytical procedures: text and methodology Q2(R1) (2005).

20.- H. Luftmann. Anal Bioanal Chem 378, 964, (2004).

21.- U. Jautz, G. Morlock. J Chromatogr A 1128, 244, (2006).

22.- G. Morlock, W. Schwack. Anal Bioanal Chem 385, 586, (2006).

23.- A. Alpmann, G. Morlock. Anal Bioanal Chem 386, 1543, (2006). 